Research Paper

\title{
Pretreatment Alkaline Phosphatase and Epstein-Barr Virus DNA Predict Poor Prognosis and Response to Salvage Radiotherapy in Patients with Nasopharyngeal Carcinoma and Metachronous Bone-Only Metastasis
}

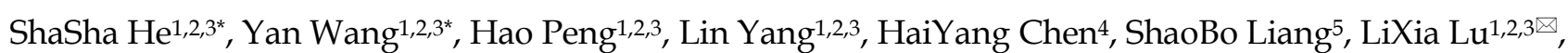
Yong Chen ${ }^{1,2,3 凶}$

1. Sun Yat-sen University Cancer Center, 651 Dongfeng Road East, Guangzhou, China;

2. State Key Laboratory of Oncology in Southern China, Guangzhou, China;

3. Collaborative Innovation Center for Cancer Medicine, Guangzhou, China;

4. The Sixth Affiliated Hospital of Sun Yat-sen University, Guangzhou, China;

5. The First Hospital of Foshan, Foshan, China.

*These authors contributed equally to this work.

$\triangle$ Corresponding authors: Yong Chen, M.D.; Lixia Lu, M.D., PhD; Sun Yat-sen University Cancer Center, State Key Laboratory of Oncology in South China; Collaborative Innovation Center for Cancer Medicine, Department of Radiation Oncology, Guangzhou 510060, People's Republic of China. Telephone: +0086-020-87343505, E-mail: chenyong@sysucc.org.cn; lulx@sysucc.org.cn.

(C) Ivyspring International Publisher. This is an open access article distributed under the terms of the Creative Commons Attribution (CC BY-NC) license (https://creativecommons.org/licenses/by-nc/4.0/). See http://ivyspring.com/terms for full terms and conditions.

Received: 2016.08.23; Accepted: 2016.10.29; Published: 2017.02.10

\begin{abstract}
Background: The bones are the most common site of distant metastasis in nasopharyngeal carcinoma (NPC). Few prognostic markers are available to guide treatment and sub-classify patients with bone metastasis. We aimed to identify the prognostic value of pretreatment serum alkaline phosphatase (ALP) and plasma Epstein-Barr virus DNA (EBV DNA) in patients with bone-only metastasis.

Methods: A total of 272 patients who developed bone-only metastases after therapy were retrospectively analyzed. Patients were categorized according to pretreatment serum ALP (< or $\geq 110$ $\mathrm{U} / \mathrm{L}$ ) and pretreatment plasma EBV DNA (< or $\geq 6,750$ copies $\left.\mathrm{ml}^{-1}\right)$. Univariate and multivariate analyses of clinical variables were performed using Cox proportional hazards regression models. Overall survival (OS) was analyzed using the Kaplan-Meier method and compared using the log-rank test.

Results: Median OS for the cohort was 34.06 months (range, 2.53-143.87 months). Multivariate Cox proportional hazard analysis verified pretreatment serum ALP and pretreatment plasma EBV DNA were independent prognostic factors for OS. In stratified survival analysis of patients with elevated pretreatment serum ALP and/or plasma EBV DNA, delivery of radiotherapy (RT) to bone metastases provided a significant $O S$ benefit compared to other therapeutic methods $(P<0.05)$.

Conclusions: This study demonstrates two important points: firstly, pretreatment serum ALP and plasma EBV DNA have prognostic value at the first diagnosis of bone-only metastasis in NPC. Secondly, radiotherapy of bone metastasis improves the prognosis of patients with elevated pretreatment serum ALP and plasma EBV DNA.
\end{abstract}

Key words: Nasopharyngeal carcinoma; bone metastasis; alkaline phosphatase; Epstein-Barr virus DNA; prognosis.

\section{Introduction}

Nasopharyngeal carcinoma (NPC) is a malignancy that is disproportionally common in eastern Asia, with the highest incidence of approximately 20 per 100,000 people per year observed among the Cantonese population in the South of China $[1,2]$. A recent epidemiological 
analysis reported NPC was related to district, age, gender, diet, lifestyle, Epstein-Barr virus (EBV) infection, genetic factors and even the type of dialect in China [3]. As it has a complicated anatomical localization, NPC is considered surgically unresectable, but is sensitive to radiotherapy and chemotherapy. Combination of radiotherapy and chemotherapy has been proven to provide survival benefits for patients with advanced stage NPC [4]. In combination with developments in radiotherapy technology and imaging modalities, these therapeutic improvements have led to a reduction in mortality among patients with NPC since the peak value of 1.45/105 in 2002 [5] .

Approximately $15-19 \%$ of all patients with NPC develop distant metastasis [6]. NPC frequently presents as metastatic disease, with a propensity for metastasizing to bone. The bones account for 50\% cases of metastasis, and metastasis to bone is associated with a poor prognosis and survival outcomes [7]. At present, the selection of radioand/or chemotherapeutic treatments for NPC are generally based on the characteristics of the primary tumor [8]. However, the current TNM staging system possesses a number of flaws, as patients with the same TNM stage display different clinical behaviors and achieve varied treatment responses and outcomes [9]. Despite the relative unreliability of the current TNM staging system, there is a lack of related research and the prognostic value of clinicopathological factors, risk assessment of different cases and the role of combined treatment in patients with bone metastasis have not been systematically defined and explored.

Currently, there is much interest in discovering prognostic markers that may help to discriminate patients and guide effective therapeutic strategies $[10,11]$.

However, the identification of reliable biomarkers associated with prognosis is a continuous challenge. Surely, there have existed some studies about the prognostic value of ALP in the patients with NPC [12] and metastastic NPC [13].

Therefore, the aim of this study was to investigate the prognostic value of serum alkaline phosphatase (ALP) and plasma Epstein-Barr virus DNA (EBV DNA) in patients with bone-only metastatic NPC in order to assist individual treatment decision-making for patients with M1 stage NPC.

\section{Materials and Methods}

\section{Patients}

Between March 2004 and October 2013, the medical records of 2,685 patients with newly-diagnosed NPC who received therapy at Sun Yat-sen University Cancer Center (SYSUCC), China, were retrospectively reviewed. Prior patient consent and approval from the Institutional Review Board of SYSUCC were obtained for the review of clinical data for research purposes. We only included patients who underwent skeletal scintigraphy and 18F-FDG PET so that metastases at diagnosis would not be missed. Patients lacking complete records on clinical and hematological variables and follow-up data were excluded. Overall, the 272/2685 (10.1\%) patients who developed bone metastasis after primary treatment (not including patients with bone metastasis at first diagnosis) were included in this analysis.

\section{Clinical stage}

For each patient, a complete medical history and physical examination were conducted, in conjunction with check-ups including serum biochemistry tests, direct fiber-optic nasopharyngoscopy, nasopharyngeal and neck magnetic resonance imaging (MRI), abdominal ultrasound, chest radiography and entire body bone scan and positron emission tomography-computed tomography (PET-CT). Plasma EBV DNA load was routinely measured before treatment using real-time quantitative PCR, as described previously [14], and the undetectable EBV signals in the sample were reported as 0 copy $/ \mathrm{mL}$. Clinical stage was classified according to the 7th edition of the International Union against Cancer/American Joint Committee on Cancer (UICC/AJCC) staging system for NPC [15].

\section{Clinical data and treatment}

The following data were recorded: clinical (age, gender, overall stage, $\mathrm{T}$ category, $\mathrm{N}$ category, radiotherapy technique, primary treatment, radiotherapy after diagnosis of metastasis), pathology (pathological pattern), and baseline laboratory data (serum alkaline phosphatase [ALP], plasma EBV DNA). Blood samples were routinely drawn at each patient's first appointment at our department (before initiation of any diagnostic or therapeutic interventions).

All patients received primary curative-intent treatment. Concurrent chemoradiotherapy with or without neoadjuvant or adjuvant chemotherapy was administered for advanced-stage disease (stage III-IVB), and either platinum-based chemotherapy or radiotherapy (2D/3DCRT/IMRT) was administered for early-stage disease or to patients that could not tolerate combined therapy.

After diagnosis of bone metastasis, we developed a treatment plan based on the general status of the patient and their illness. Treatment 
modalities for bone metastasis varied depending on the metastatic time order, site and number of metastases. Radiotherapy was prescribed for localized bone metastatic lesions to cure or relieve pain; chemotherapy was added for palliative purposes. The radiotherapy doses/fractions and chemotherapy regimens were selected individually for each patient. In our study, the salvage radiotherapy technique for all patients were intensity modulated radiotherapy (IMRT), and with a dose of 30Gy/10Fractions. Chemotherapy was the primary treatment, the most widely-used regimen was platins (cisplatin, carboplatin) or platins in association with docetaxel, paclitaxel or gemcitabine. The number of chemotherapy cycles was based on the patient's physical condition. In total, 120/272 (44.1\%) patients received systemic chemotherapy only to treat bone metastases; 100 (36.8\%) chemoradiotherapy; 35 (12.9\%) local radiotherapy only; and 17 (6.2\%) symptomatic therapy only.

\section{Follow-up}

Follow-up evaluations were performed every 3 months within the first 3 years, 6 months for the following 2 years and annually thereafter until death. The follow-up duration was calculated from the first day of therapy to the day of death or last examination. The last follow-up time was May, 2016. The study end-point was overall survival (OS), defined as the time from the date of first diagnosis to the date of death or last known date alive. Disease-free interval (DFI) was measured as the duration between the end of primary treatment and the date of first diagnosis of bone-only metastasis, which was diagnosed based on symptoms and a bone scan.

\section{Statistical analysis}

We established a cut-off value for EBV DNA by applying receiver operating characteristics (ROC) analysis. The Chi-square test was used to analyze the correlations between DFI and clinicopathological features. Univariate and multivariate analysis of clinical variables were performed using Cox proportional hazards regression models. Variables in the model included gender, age, overall stage, $\mathrm{T}$ category, N category, ALP, EBV DNA, radiotherapy after metastasis, vertebral metastasis, number of metastatic sites and DFI. Survival rates were plotted against time using the Kaplan-Meier method, and log-rank testing was used to compare the differences between the curves. Statistical analysis was performed using IBM SPSS statistical program package, version 22.0, IBM Corp. All statistical tests were two-sided; a $P$ value $<0.05$ was considered statistically significant.

\section{Results}

\section{Patient characteristics}

Of the 272 patients with NPC who developed bone-only metastasis after treatment, 258 (94.9\%) were male and $14(5.1 \%)$ were female, and 17 (6.2\%) were initially diagnosed with early stage disease (Stage I-II) and 255 (93.8\%) with advanced stage NPC (Stage III-IVB). The baseline characteristics of the patients are presented in Table 1 . The median follow-up time was 34.06 months (range, 2.53-143.87 months). At last follow-up, 132/272 (48.5\%) patients had died. The 3-year OS rate for the entire cohort was $62.9 \%$.

Table 1. Baseline characteristics of the 272 patients with nasopharyngeal cancer

\begin{tabular}{ll}
\hline Characteristics & $n \mathbf{( \% )}$ \\
\hline Total & $272(100)$ \\
Gender & \\
Male & $258(94.9)$ \\
Female & $14(5.1)$ \\
Age (years) & \\
$<45$ & $140(51.5)$ \\
$\geq 45$ & $132(48.5)$ \\
Overall stage ${ }^{\dagger}$ & \\
I+II & $17(6.2)$ \\
III+IVB & $255(93.8)$ \\
T category ${ }^{\dagger}$ & \\
T1-2 & $63(23.2)$ \\
T3-4 & $209(76.8)$ \\
N category ${ }^{\dagger}$ & \\
N0-1 & $81(46.1)$ \\
N2-3 & $191(53.9)$ \\
Primary treatment & \\
Radiotherapy alone & $71(29.8)$ \\
Chemoradiotherapy & $201(70.2)$ \\
Vertebral metastasis & \\
Absent & $99(36.4)$ \\
Present & $173(63.6)$ \\
Number of metastatic sites &
\end{tabular}

Abbreviations: $\mathrm{T}=$ tumor; $\mathrm{N}=$ node; ${ }^{\dagger}$ ) According to the 7 th edition of the AJCC/UICC staging system.

\section{Pretreatment serum ALP}

Median pretreatment ALP was $74.25 \mathrm{U} / \mathrm{L}$, the cut-off value for ALP was defined as the upper limit of normal (ULN) value. In total, 250/272 (91.9\%) patients were in the non-elevated ALP (NE-ALP) group (ALP < $110 \mathrm{U} / \mathrm{L}$ ) and $22(8.1 \%)$ were in the elevated ALP (E-ALP) group (ALP $\geq 110 \mathrm{U} / \mathrm{L}$ ). In univariate analysis, 3-year OS was $65.1 \%$ for the NE-ALP group vs. $33.6 \%$ for the E-ALP group $(P=$ 0.001, Figure 1A). 
A

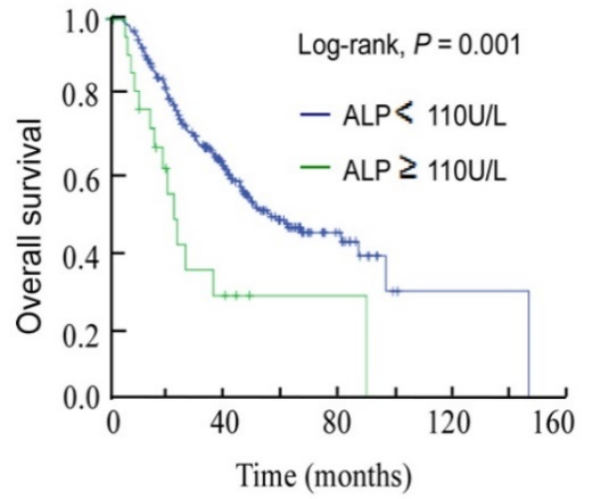

B

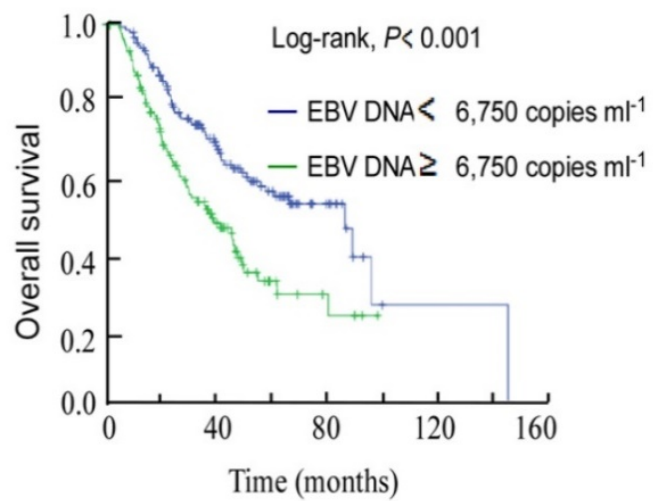

Figure 1. Kaplan-Meier overall survival curves for the 272 patients with nasopharyngeal cancer stratified by (A) pretreatment serum ALP and (B) pretreatment plasma EBV DNA.

\section{Pretreatment plasma EBV DNA}

Based on ROC curve analysis for OS, the pretreatment plasma EBV DNA cut-off value was identified as 6,750 copies $\mathrm{ml}^{-1}$. Univariate analysis showed the 3-year OS rate for the high EBV DNA group ( $\geq 6,750$ copies $\mathrm{ml}^{-1}$ ) was $51.8 \%$ compared to $71.5 \%$ for the low DNA group $\left(<6,750\right.$ copies $\mathrm{ml}^{-1}, P<$ 0.001; Figure 1B).

\section{Association between clinical variables and DFI and univariate analysis}

The median DFI for this cohort of 272 patients who developed bone-only metastasis was 13 months (range, 1.03-86.07 months). Thus, using 13 months as the cut-off value for DFI, we found that the subgroups of patients with shorter DFI presented a more advanced $\mathrm{T}$ category and N category NPC (T3-4, N2-3) than patients with longer DFI, with $P$-values of 0.015 and 0.042 , respectively. Also, there are more patients with high pretreatment ALP $(P=0.046)$ and EBV DNA $(P=0.029$; Table 2$)$ in shorter DFI group than in longer DFI group. In univariate analysis, the 3-year OS rates for patients with high DFI and low DFI were $77.9 \%$ and $39.8 \%$, respectively $(P<0.001$, Figure 2A).

\section{Prognostic value of integrating serum ALP and plasma EBV DNA}

Using pretreatment serum ALP and plasma EBV DNA, we classified the 272 patients into three groups: group A, NE-ALP and low EBV DNA; group B, NE-ALP and high EBV DNA or E-ALP and low EBV DNA; and group C, E-ALP and high EBV DNA. The 3 -year OS rates for these three groups were $74 \%$ vs. $50.9 \%$ vs. $33.8 \%$, respectively ( $P<0.001$, Figure $2 \mathrm{~B})$.

In addition, we further assessed OS for each of these groups to assess which subsets of patients benefited from local radiotherapy after developing bone metastasis. Patients whose metastases were treated with radiotherapy achieved longer OS than those who did not receive radiotherapy in both group $\mathrm{B}(P<0.001$, Figure 2D) and group C $(P=0.048$, Figure $2 \mathrm{E})$, but not in group $\mathrm{A}(P=0.230$, Figure $2 \mathrm{C})$. Moreover, radiotherapy to the metastases provided a benefit in terms of OS in analysis of the entire group $(P<0.001$, Figure 2F).

Table 2. Association between clinical variables and the disease-free interval (DFI) to bone-only metastases

\begin{tabular}{|c|c|c|c|}
\hline & DFI & & \\
\hline Clinical variable & $<13$ months & $\geq 13$ months & $P^{*}$ \\
\hline Gender & & & 0.286 \\
\hline Male & $111(96.5)$ & 147 (93.6) & \\
\hline Female & $4(3.5)$ & $10(6.4)$ & \\
\hline Age (years) & & & 0.303 \\
\hline$<45$ & $55(47.8)$ & $85(54.1)$ & \\
\hline$\geq 45$ & $60(52.2)$ & $72(45.9)$ & \\
\hline Overall stage & & & 0.358 \\
\hline $\mathrm{I}+\mathrm{II}$ & $9(7.8)$ & $8(5.1)$ & \\
\hline III+IVB & $106(92.2)$ & $149(94.9)$ & \\
\hline T category & & & 0.015 \\
\hline T1-2 & $35(30.4)$ & $28(17.8)$ & \\
\hline T3-4 & $80(69.6)$ & $129(28.2)$ & \\
\hline$N$ category & & & 0.042 \\
\hline N0-1 & $27(23.5)$ & $54(34.4)$ & \\
\hline $\mathrm{N} 2-3$ & $88(76.5)$ & $103(65.6)$ & \\
\hline Radiotherapy technique & & & 0.104 \\
\hline 2DRT/3DCRT & $29(25.2)$ & $54(34.4)$ & \\
\hline IMRT & $86(74.8)$ & $103(65.6)$ & \\
\hline Primary treatment & & & 0.164 \\
\hline Radiotherapy & $35(30.4)$ & $36(22.9)$ & \\
\hline Chemoradiotherapy & $80(69.6)$ & 121(77.1) & \\
\hline Pretreatment ALP $(U / L)$ & & & 0.046 \\
\hline$<110$ & $102(88.7)$ & $148(94.3)$ & \\
\hline$\geq 110$ & $13(11.3)$ & $9(5.7)$ & \\
\hline Pretreatment EBV DNA (copies $\mathrm{ml}^{-1}$ ) & & & 0.029 \\
\hline$<6,750$ & $52(45.2)$ & $92(58.6)$ & \\
\hline$\geq 6,750$ & $63(54.8)$ & $65(41.4)$ & \\
\hline
\end{tabular}

Abbreviations: $\mathrm{DFI}=$ disease-free interval; $2 \mathrm{DRT}=$ two-dimensional radiotherapy; 3DCRT $=$ three-dimensional conformal radiotherapy; IMRT = intensity-modulated radiation therapy; $\mathrm{LDH}=$ alkaline phosphatase.

* Chi-square test. 
A

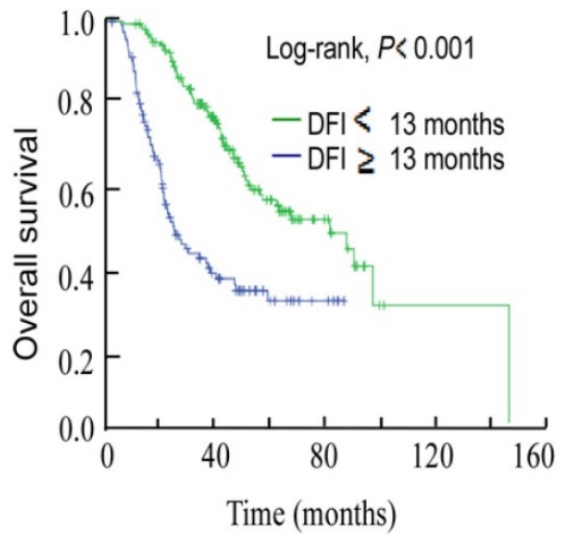

C
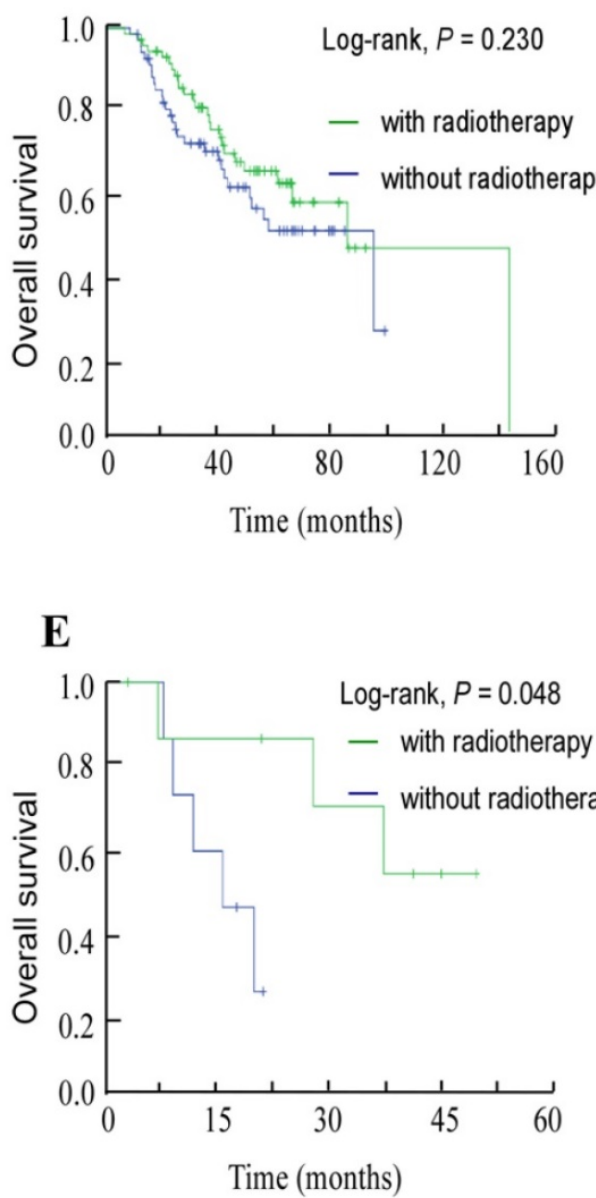

B

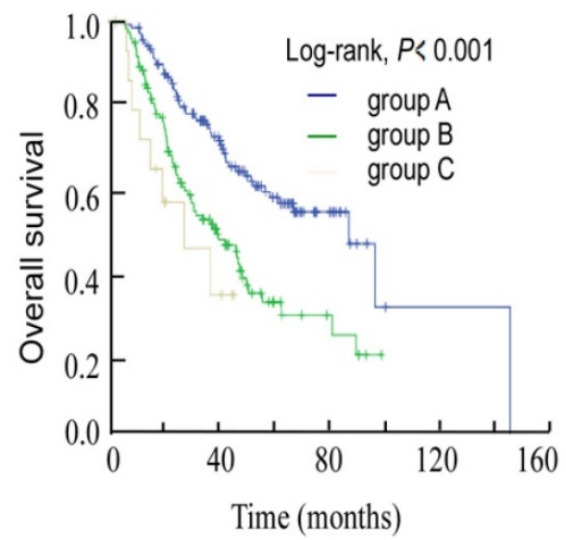

D
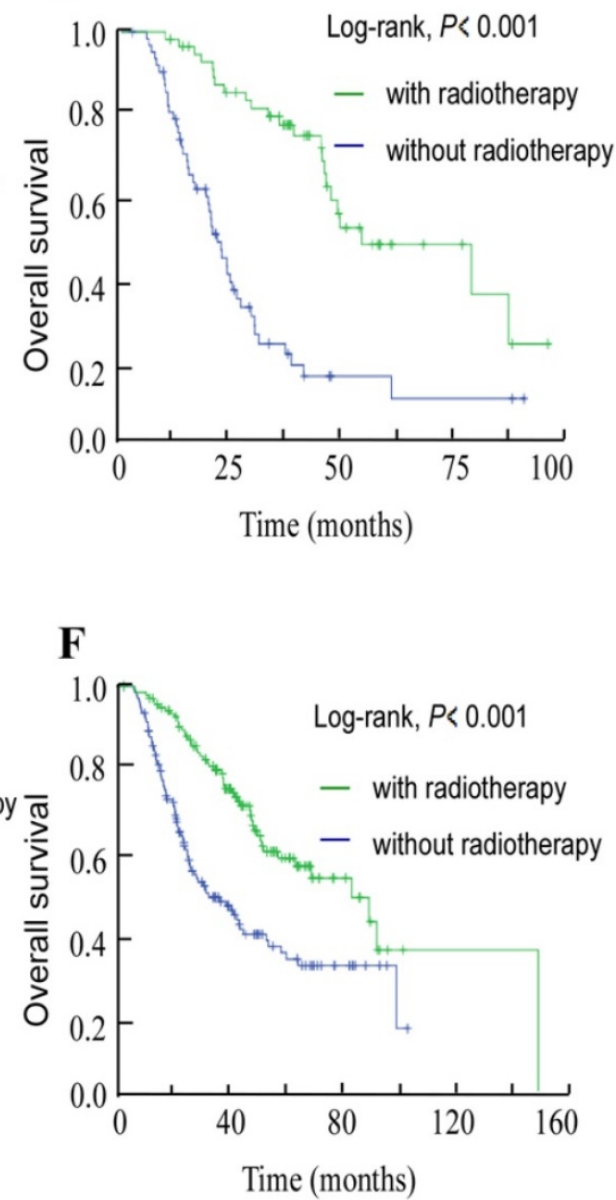

Figure 2. Kaplan-Meier overall survival curves. (A) Overall survival curves for the 272 patients with nasopharyngeal cancer stratified by the disease-free interval (DFI) to bone-only metastasis. (B) Overall survival curves for the 272 patients with nasopharyngeal cancer stratified as group A, non-elevated ALP (NE-ALP) and low EBV DNA; group B, NE-ALP and high EBV DNA or elevated ALP (E-ALP) and low EBV DNA; and group C, E-ALP and high EBV DNA. (C) Overall survival curves for group $A$ stratified by radiotherapy after metastasis; $(D)$ group $B$ stratified by radiotherapy after metastasis; $(E)$ group $C$ stratified by radiotherapy after metastasis; and $(F)$ the entire cohort of 272 patients stratified by radiotherapy after metastasis.

\section{Cox multivariate analysis for OS}

Univariate analysis indicated $\mathrm{N}$ category, pretreatment serum ALP, pretreatment EBV DNA, primary treatment, radiotherapy after metastasis, vertebral metastasis, the number of metastatic sites and DFI were significant predictors of OS. Multivariate analysis to adjust for these factors revealed pretreatment serum ALP (HR 1.720, 95\% CI 1.199-2.467, $P=0.003$ ) and pretreatment plasma EBV DNA (HR 1.635, 95\% CI 1.131-2.363, $P=0.009$ ) were 
independent prognostic factors for OS. The other independent significant prognostic factors were $\mathrm{N}$ category, radiotherapy after metastasis, vertebral metastasis, the number of metastatic sites and DFI (Table 3).

Table 3. Univariate and multivariate analyses for overall survival (OS)

\begin{tabular}{|c|c|c|c|c|c|c|}
\hline \multirow[t]{2}{*}{ Characteristics } & \multicolumn{3}{|c|}{ Univariate analysis } & \multicolumn{3}{|c|}{ Multivariate analysis } \\
\hline & HR & $95 \% \mathrm{CI}$ & $P$ & HR & 95\% CI & $P$ \\
\hline \multicolumn{7}{|l|}{ Gender } \\
\hline $\begin{array}{l}\text { Female vs. } \\
\text { male }\end{array}$ & 1.275 & $0.62-2.623$ & 0.508 & & & \\
\hline \multicolumn{7}{|l|}{ Age (years) } \\
\hline$\geq 45$ vs. $<45$ & 1.199 & $0.848-1.694$ & 0.304 & & & \\
\hline \multicolumn{7}{|l|}{ Overall stage } \\
\hline $\begin{array}{l}\text { III + IVB vs. I + } \\
\text { II }\end{array}$ & 1.373 & $0.641-2.943$ & 0.415 & & & \\
\hline \multicolumn{7}{|l|}{$\mathrm{T}$ category } \\
\hline T3-4 vs. T1-2 & 1.029 & $0.684-1.549$ & 0.891 & & & \\
\hline \multicolumn{7}{|l|}{$\mathrm{N}$ category } \\
\hline N2-3 vs. N0-1 & 1.913 & $1.572-2.326$ & $<0.001$ & 1.467 & $1.175-1.832$ & 0.001 \\
\hline \multicolumn{7}{|l|}{$\begin{array}{l}\text { Pretreatment } \\
\text { ALP }(\mathrm{U} / \mathrm{L})\end{array}$} \\
\hline$\geq 110$ vs. $<110$ & 2.517 & $1.438-4.406$ & 0.001 & 1.720 & $1.199-2.467$ & 0.003 \\
\hline \multicolumn{7}{|l|}{$\begin{array}{l}\text { Pretreatment } \\
\text { EBV DNA } \\
\left(\text { copies } \mathrm{ml}^{-1} \text { ) }\right.\end{array}$} \\
\hline $\begin{array}{l}\geq 6,750 \text { vs. }< \\
6,750\end{array}$ & 1.933 & $1.362-2.745$ & $<0.001$ & 1.635 & $1.131-2.363$ & 0.009 \\
\hline \multicolumn{7}{|l|}{$\begin{array}{l}\text { IMRT after } \\
\text { metastasis }\end{array}$} \\
\hline No vs. yes & 1.470 & $1.009-2.143$ & 0.045 & 1.578 & $1.077-2.314$ & 0.019 \\
\hline \multicolumn{7}{|l|}{$\begin{array}{l}\text { Vertebral } \\
\text { metastasis }\end{array}$} \\
\hline $\begin{array}{l}\text { Present vs. } \\
\text { absent }\end{array}$ & 2.269 & $1.531-3.363$ & $<0.001$ & 1.997 & $1.321-3.020$ & 0.001 \\
\hline \multicolumn{7}{|l|}{$\begin{array}{l}\text { Number of } \\
\text { metastatic sites }\end{array}$} \\
\hline$\geq 3$ vs. $<3$ & 1.539 & $1.078-2.198$ & 0.018 & 1.531 & $1.056-2.220$ & 0.025 \\
\hline \multicolumn{7}{|l|}{ DFI (months) } \\
\hline$\geq 13$ vs. $<13$ & 2.824 & $1.983-4.023$ & $<0.001$ & 2.868 & $1.987-4.139$ & 0.001 \\
\hline
\end{tabular}

\section{Discussion}

In this study, we systematically evaluated OS, prognostic factors (especially ALP and EBV DNA), and post-metastasis treatment modalities for patients with NPC who develop bone-only metastasis as the first failure after primary treatment. A number of noteworthy findings emerged from this retrospective analysis. First, both elevated pretreatment serum ALP and pretreatment plasma EBV DNA were associated with significantly shorter DFI and poorer OS. Second, Cox multivariate analysis indicated $\mathrm{N}$ category, pretreatment serum ALP, pretreatment plasma EBV DNA, primary treatment, radiotherapy after metastasis, vertebral metastasis, the number of metastatic sites and DFI were independent prognostic factors for OS. Third, delivery of local radiotherapy to the sites of metastasis may provide an OS benefit compared to other treatments in patients with bone metastasis.

It has been previously reported that patients with NPC have a high incidence of early bone metastasis, with the spine being the most common site of skeletal metastasis, accounting for $66.0 \%$ (of 206 cases) [16]. In this cohort, spinal involvement was associated with poorer OS (HR, 1.997, 95\% CI $1.199-2.467, P=0.001)$. These results are similar to a previous study (HR: 1.91; 95\% CI: 1.33-2.74) [11]. It is well-recognized that the diagnostic ability of imaging for early bone metastatic lesions is far from sensitive, especially when the tumor cells are mainly confined to the bone marrow [17]. In this study, patients were diagnosed with bone metastasis via a number of imaging technologies, each of which have disadvantages: bone scans have a low specificity and high false-positive rate, CT requires the use of radioisotopes, PET/CT is expensive and MRI also suffers from limitations include partial-volume effects. Moreover, skeletal-related events (SREs) occur non-specifically: one study reported that $12.1 \%$ of patients with bone metastasis did not have SREs [18]. Thus, the imaging technologies employed for diagnosis of bone metastasis have inherent limitations that may lead to a delay in diagnosis, especially in patients without the symptoms of SREs.

In recent years, a number of serum markers have been reported to have prognostic value in patients with NPC and bone metastasis, including lactic dehydrogenase (LDH) and ALP.

Elevated serum ALP has been associated with the presence of bone, liver and other diseases [19]. ALP is divided into four isozymes named after the site of tissue expression: intestinal ALP, placental ALP, germ cell ALP and liver/bone/kidney ALP [20]. Although these ALPs are present in many tissues and have been investigated for a number of years, relatively little is known about their functions. ALP is a by-product of osteoblast activity, which is related to bone metabolism; therefore, elevated ALP indicates that bone formation could be occurring. Metabolic disorders of bone disease contribute to ALP elevation $[21,22]$. However, elevated ALP can be due to diseases in several other organs. Therefore, we excluded patients who presented evidence of diseases known to cause abnormal ALP levels. A recent review explored the impact of serum ALP on the clinical response and survival outcomes of patients with skeletal metastases in NPC [13]. Likewise, our results confirmed high serum ALP level before treatment predicted poor OS in patients with NPC who developed bone-only metastasis (HR 1.720, 95\% CI 1.199-2.467, $P=0.003$ ). 
Clinical use of EBV in NPC continues to increase, with quantitative analyses of circulating EBV DNA used for population screening, prognostication and disease surveillance [23]. At present, randomized trials are in progress to investigate the role of EBV DNA in order to stratify patients for tailored treatment. Patients with high plasma EBV DNA require more intense treatment [24, 25]. However, the prognostic value of EBV DNA in patients with bone-only metastasis has not been explored. Thus, we preformed stratified analysis by pretreatment EBV DNA. ROC curve analysis for OS determined the cut-off value for EBV DNA should be 6,750 copies $\mathrm{ml}^{-1}$, which is much higher than that of the previously published cut-off values selected for analysis in patients with non-metastatic NPC treated in our department [26, 27]. According to our mutilvariate analysis, pretreatment plasma EBV DNA were independent prognostic factors for OS (HR 1.635, 95\% CI 1.131-2.363, $P=0.009$ ) in patients with skeletal metastasis.

After confirming the prognostic value of pretreatment serum ALP and pretreatment plasma EBV DNA, we categorized the patients into three subgroups. Kaplan-Meier curves revealed patients with elevated pretreatment ALP and high pretreatment EBV DNA achieved the poorest OS. In addition, further exploratory analysis was conducted to identify whether OS may differ in the subgroups who received radiotherapy or other treatments after metastasis. Remarkably, local radiotherapy for bone metastases provided an OS benefit over the treatment modalities that without radiotherapy in the subgroups of patients with elevated pretreatment serum ALP, high plasma pretreatment EBV DNA, or both elevated ALP and high EBV DNA. These results indicate that the combination of high pretreatment serum ALP and high pretreatment plasma EBV DNA is able to discriminate patients for whom radiotherapy to the metastases will result in a better prognosis compared to no radiotherapy or other treatments.

Currently, systemic chemotherapy is the primary palliative treatment for patients with NPC who develop metastases, with numerous confirmations of its efficacy published [28]. However, median survival for patients who develop metastases is short. When combined with locoregional radiotherapy, systemic chemotherapy improves the survival, disease control and quality of life of patients with metastatic NPC $[1,29]$. However, it has not been confirmed whether locoregional radiotherapy targeting the sites of bone metastasis affects the survival of patients with bone-only metastatic NPC. In this study, patients underwent chemotherapy, radiotherapy, targeted therapy, combined use of bisphosphonates, or only symptomatic therapy; $135 / 272(49.6 \%)$ patients received locoregional radiotherapy. Of the entire cohort, univariate and multivariate analyses demonstrated that radiotherapy after metastasis improved OS, and confirmed radiotherapy after metastasis was an independent significant prognostic factor for OS.

One limitation of this study that cannot be neglected is that the application of various adjuvant therapeutic methods may confuse the results; and moreover, the varied therapeutic protocols may also have led to diverse survival outcomes. Thus, randomized studies are necessary to authenticate the prognostic value of pretreatment serum ALP and pretreatment plasma EBV DNA, confirm the survival benefit of locoregional radiotherapy in patients with NPC who develop bone-only metastases, and further exploration of adjuvant therapies is warranted. Additionally, the quality of life of patients with metastatic NPC has received little investigation, and any future research to validate this study should consider the effect of such treatments on the patients' quality of life.

\section{Conclusions}

Several prognostic factors for OS were identified for patients with NPC who developed bone-only metastases. Using pretreatment serum ALP and plasma EBV DNA, as well as other clinicopathological factors, patients with bone-only metastases can be more effectively stratified into risk groups to guide individualized treatment. Additionally, radiotherapy after metastasis significantly improved OS, especially in patients with high pretreatment serum ALP and/or plasma EBV DNA, and radiotherapy after metastasis was an independent significant prognostic factor for OS.

\section{Acknowledgments}

This work was supported by the Natural Science Foundation of Guangdong Province, China [No. 2016A020215083]. The funding agency had no role in the study design, data collection and analysis, decision to publish, or preparation of the manuscript.

\section{Competing Interests}

The authors have declared that no competing interest exists.

\section{References}

1. Wei WI, Sham JS. Nasopharyngeal carcinoma. Lancet (London, England). 2005; 365: 2041-54.

2. $\mathrm{Li} \mathrm{CC}, \mathrm{Yu} \mathrm{MC}$, Henderson BE. Some epidemiologic observations of nasopharyngeal carcinoma in Guangdong, People's Republic of China. National Cancer Institute monograph. 1985; 69: 49-52. 
3. Cao SM, Simons MJ, Qian CN. The prevalence and prevention of nasopharyngeal carcinoma in China. Chinese journal of cancer. 2011; 30: 114-9.

4. Huncharek M, Kupelnick B. Combined chemoradiation versus radiation therapy alone in locally advanced nasopharyngeal carcinoma: results of a meta-analysis of 1,528 patients from six randomized trials. American journal of clinical oncology. 2002; 25: 219-23.

5. Xu ZX, Lin ZX, Fang JY, Wu KS, Du PL, Zeng Y, et al. Mortality Characteristic and Prediction of Nasopharyngeal Carcinoma in China from 1991 to 2013. Asian Pacific journal of cancer prevention : APJCP. 2015; 16: 6729-34.

6. Razak AR, Siu LL, Liu FF, Ito E, O'Sullivan B, Chan K. Nasopharyngeal carcinoma: the next challenges. European journal of cancer (Oxford, England : 1990). 2010; 46: 1967-78.

7. Sham JS, Choy D, Choi PH. Nasopharyngeal carcinoma: the significance of neck node involvement in relation to the pattern of distant failure. The British journal of radiology. 1990; 63: 108-13.

8. Greene FL, Sobin LH. The staging of cancer: a retrospective and prospective appraisal. CA: a cancer journal for clinicians. 2008; 58: 180-90.

9. Au JS, Law CK, Foo W, Lau WH. In-depth evaluation of the AJCC/UICC 1997 staging system of nasopharyngeal carcinoma: prognostic homogeneity and proposed refinements. International journal of radiation oncology, biology, physics. 2003; 56: 413-26.

10. Lu T, Guo Q, Cui X, Chen Z, Lin S, Xu L, et al. Prognostic Evaluation of Nasopharyngeal Carcinoma with Bone-Only Metastasis after Therapy. Yonsei medical journal. 2016; 57: 840-5.

11. Shen L, Dong J, Li S, Wang Y, Dong A, Shu W, et al. M1 stage subdivision and treatment outcome of patients with bone-only metastasis of nasopharyngeal carcinoma. The oncologist. 2015; 20: 291-8.

12. Xie Y, Wei ZB, Duan XW. Prognostic value of pretreatment serum alkaline phosphatase in nasopharyngeal carcinoma. Asian Pacific journal of cancer prevention : APJCP. 2014; 15: 3547-53.

13. Jin $Y$, Yuan MO, Chen JQ, Zhang YP. Serum alkaline phosphatase predicts survival outcomes in patients with skeletal metastatic nasopharyngeal carcinoma. Clinics (Sao Paulo, Brazil). 2015; 70: 264-72.

14. Shao JY, Zhang Y, Li YH, Gao HY, Feng HX, Wu QL, et al. Comparison of Epstein-Barr virus DNA level in plasma, peripheral blood cell and tumor tissue in nasopharyngeal carcinoma. Anticancer research. 2004; 24: 4059-66.

15. Li J, Zou X, Wu YL, Guo JC, Yun JP, Xu M, et al. A comparison between the sixth and seventh editions of the UICC/AJCC staging system for nasopharyngeal carcinoma in a Chinese cohort. PloS one. 2014; 9: e116261.

16. Sundram FX, Chua ET, Goh AS, Toh HJ, Khor TH, Chua EJ. Bone scintigraphy in nasopharyngeal carcinoma. Clinical radiology. 1990; 42: 166-9.

17. Algra PR, Bloem JL, Tissing H, Falke TH, Arndt JW, Verboom LJ. Detection of vertebral metastases: comparison between MR imaging and bone scintigraphy. Radiographics : a review publication of the Radiological Society of North America, Inc. 1991; 11: 219-32.

18. Cetin K, Christiansen CF, Jacobsen JB, Norgaard M, Sorensen HT. Bone metastasis, skeletal-related events, and mortality in lung cancer patients: a Danish population-based cohort study. Lung cancer (Amsterdam, Netherlands). 2014; 86: 247-54.

19. Epstein E, Kiechle FL, Artiss JD, Zak B. The clinical use of alkaline phosphatase enzymes. Clinics in laboratory medicine. 1986; 6: 491-505.

20. Sharma U, Pal D, Prasad R. Alkaline phosphatase: an overview. Indian journal of clinical biochemistry : IJCB. 2014; 29: 269-78.

21. Ardeshirpour L, Cole DE, Carpenter TO. Evaluation of bone and mineral disorders. Pediatric endocrinology reviews : PER. 2007; 5 Suppl 1: 584-98.

22. Li G, Gao J, Tao YL, Xu BQ, Tu ZW, Liu ZG, et al. Increased pretreatment levels of serum LDH and ALP as poor prognostic factors for nasopharyngeal carcinoma. Chinese journal of cancer. 2012; 31: 197-206.

23. Leung SF, Chan AT, Zee B, Ma B, Chan LY, Johnson PJ, et al. Pretherapy quantitative measurement of circulating Epstein-Barr virus DNA is predictive of posttherapy distant failure in patients with early-stage nasopharyngeal carcinoma of undifferentiated type. Cancer. 2003; 98: 288-91.

24. An X, Wang FH, Ding PR, Deng L, Jiang WQ, Zhang L, et al. Plasma Epstein-Barr virus DNA level strongly predicts survival in metastatic/recurrent nasopharyngeal carcinoma treated with palliative chemotherapy. Cancer. 2011; 117: 3750-7.

25. Chen WH, Tang LQ, Guo SS, Chen QY, Zhang L, Liu LT, et al. Prognostic Value of Plasma Epstein-Barr Virus DNA for Local and Regionally Advanced Nasopharyngeal Carcinoma Treated With Cisplatin-Based Concurrent Chemoradiotherapy in Intensity-Modulated Radiotherapy Era. Medicine. 2016; 95: e2642.

26. Peng $\mathrm{H}$, Guo $\mathrm{R}$, Chen $\mathrm{L}$, Zhang $\mathrm{Y}$, Li WF, Mao YP, et al. Prognostic Impact of Plasma Epstein-Barr Virus DNA in Patients with Nasopharyngeal Carcinoma Treated using Intensity-Modulated Radiation Therapy. Scientific reports. 2016; 6: 22000.

27. Tang LQ, Chen QY, Guo SS, Chen WH, Li CF, Zhang L, et al. The impact of plasma Epstein-Barr virus DNA and fibrinogen on nasopharyngeal carcinoma prognosis: an observational study. British journal of cancer. 2014; 111: 1102-11.

28. Li AC, Xiao WW, Shen GZ, Wang L, Xu AA, Cao YQ et al. Distant metastasis risk and patterns of nasopharyngeal carcinoma in the era of IMRT: long-term results and benefits of chemotherapy. Oncotarget. 2015; 6: 24511-21.

29. Chen MY, Jiang R, Guo L, Zou X, Liu O, Sun R, et al. Locoregional radiotherapy in patients with distant metastases of nasopharyngeal carcinoma at diagnosis. Chinese journal of cancer. 2013; 32: 604-13. 